\title{
UM RELATO DE EXPERIÊNCIA SOBRE A SEXUALIDADE NA TERCEIRA IDADE
}

\begin{abstract}
Renata Fernandes de Medeiros Lima1', ORCID ID 0000-0003-2246-2823; Gabriela Freitas Duarte², ORCID ID 0000-0003-3755-1474; Gabriela Martins Silva33, ORCID ID 0000-0001-8895-4471; Louise Lopes Barros4, ORCID ID 0000-0003-4416-697X; Ticianne Nunes de Miranda Bento5, ORCID ID 0000-0001-7794-6505; Maria Auxiliadora Lamas Fernandes de Oliveira6, ORCID ID 0000-0003-1345-7459
\end{abstract}

\section{FILIAÇÃO}

(1) Universidade Potiguar, Acadêmica de Medicina

(2) Universidade Potiguar, Acadêmica de Medicina

(3) Universidade Potiguar, Acadêmica de Medicina

(4) Universidade Potiguar, Acadêmica de Medicina

(5) Universidade Potiguar, Acadêmica de Medicina

(6) Universidade Federal do Rio Grande do Norte, Pós-graduada em Gastroenterologia pela Fundação Carlos Chagas no Hospital da Beneficência Portuguesa, Pós-graduada em Endoscopia Digestiva Alta pelo Hospital Central da Aeronáutica do Estado do Rio de Janeiro, Especialista em Endoscopia Digestiva pela SOBED.

\section{AUTOR CORRESPONDENTE}

Renata Fernandes de Medeiros Lima; renatamedeiros30@gmail.com; Av. Rodrigues Alves, 410, 59020-058 Petrópolis, Apto.2000; Universidade Potiguar

\section{MENSAGENS-CHAVE}

As concepções culturais, pautadas no preconceito, retiraram do idoso o direito de vivenciar em plenitude a sua sexualidade.

A discussão abriu portas para uma perspectiva ampla, em que há acolhimento às mudanças vivenciadas pela pessoa idosa.

A abordagem ao tema ressignificou o sentido da sexualidade na terceira idade, desmistificando os obstáculos impostos pela sociedade.

A experiência impactou positivamente, promovendo uma reflexão coletiva referente à sexualidade e voltada à saúde do idoso.

\footnotetext{
RESUMO

INTRODUÇÃO: A sexualidade na terceira idade é um tema ainda bastante negligenciado e permeado de preconceitos, o que acarreta consequências negativas para esta população, visto que, frequentemente, o idoso não tem acesso fácil à informação segura sobre as transformações biopsicossociais na senescência e suas repercussões, desconhecendo, geralmente, os benefícios de se vivenciar plenamente a sexualidade. RELATO DE EXPERIÊNCIA: Assim, a ação "Sexualidade tem prazo de validade? Uma abordagem na terceira idade" ocorreu no dia 25 de novembro de 2020, com o princípio de desmistificar tabus e estigmas sociais acerca da vivência e expressão da sexualidade pelo idoso e fomentar discussão responsável, por meio de abordagem multiprofissional, para a construção de uma nova perspectiva no acolhimento e na promoção de saúde desse grupo. Para tanto, foram utilizados o Instagram e o WhatsApp como ferramentas de divulgação do evento. Nesse contexto, as palestras aconteceram virtualmente, com espaço para debate e elucidação de questionamentos sobre o tema, além da aplicação de questionários antes e depois, para avaliação do impacto. DISCUSSÃO: Diante disso, o compartilhamento de informações sobre o sexo saudável na terceira idade é essencial para vivenciar essa fase da vida com plenitude e segurança,
} 
sem crenças limitantes. Ademais, a abordagem sensível influencia diretamente na disseminação do conhecimento e os ambientes que permitem a partilha de experiências mostram-se bastante eficazes no contexto de autocuidado e identificação entre os ouvintes. Conclusão: Isso posto, entende-se que ainda é preciso intensificar esse processo educativo, entretanto cada avanço ressignifica a concepção de saúde entre as pessoas. Nessa perspectiva, a atividade impactou positivamente os participantes, haja vista discutir sobre as principais problemáticas acerca da sexualidade na terceira idade, abordando desde a dificuldade de acesso aos conteúdos de saúde até o processo de vivência e expressão desse envelhecimento, tendo como foco a qualidade de vida e a liberdade sexual.

\title{
PALAVRAS-CHAVE: Idosos; Promoção do Bem Estar; Comportamento Sexual; Sexo
}

\begin{abstract}
INTRODUCTION: Sexuality in the elderly is still a neglected theme, permeated by prejudice, which has negative consequences for this population, since the elderly often do not have easy access to safe information about the biopsychosocial changes in senescence and its repercussions, and are generally unaware of the benefits of fully experiencing sexuality. EXPERIENCE REPORT: The event "Does sexuality have an expiration date? An approach in the elderly" occurred on November 25th, 2020, with the principle of demystifying taboos and social stigmas about the experience and expression of sexuality by the elderly and fostering responsible discussion, through a multidisciplinary approach, for the construction of a new perspective in the reception and health promotion of this group. Instagram and WhatsApp were used as tools to publicize the event. In this context, the lectures took place virtually, with room for debate and clarification of questions on the subject, in addition to the application of questionnaires before and after in order to assess the impact. DISCUSSION: Therefore, sharing information about healthy sex in old age is essential to live this phase of life fully and safely, without limiting beliefs. Also, the sensitive approach directly influences the dissemination of knowledge, and environments that allow the sharing of experiences are effective in the context of self-care and identification among listeners. CONCLUSION: That said, it is understood that it is still necessary to intensify this educational process, however, each advance gives new meaning to the concept of health among people. The activity had a positive impact on the participants, as it discussed the main problems about sexuality in old age, from the difficulty of access to health content to the process of experience and expression of aging, focusing on quality of life and sexual freedom.
\end{abstract}

\section{KEYWORDS: Elderly; Health Promotion; Sexual Behavior; Sex}

\section{INTRODUÇÃO}

A sexualidade deve ser compreendida como intrínseca a todo o indivíduo, a qualquer momento de sua vida, considerada singular a cada pessoa (1). Nesse sentido, tendo um olhar voltado para os idosos, vê-se que estereótipos de uma velhice assexuada permanecem no imaginário social (2), logo, apesar da notória importância para o bem-estar, a qualidade de vida e a saúde dos idosos, abordar esse assunto é algo, às vezes, complicado, visto que o tabu está muito presente. Sendo assim, encontrar uma roda de conversa ou uma oportunidade de troca de experiência e informação confiável é algo escasso.

Dessa forma, visando proporcionar dignidade e uma vida mais satisfatória para essa parte da população, foi realizada uma roda de conversa com o tema "Sexualidade tem prazo de validade? Uma abordagem na terceira idade", com a finalidade de tentar romper crenças limitantes no que tange à abordagem do assunto e informar a população idosa sobre riscos, benefícios e mudanças anatômicas e fisiológicas dessa fase, abordando a perspectiva de que envelhecer é algo biológico, e isso não é sinônimo do fim da vida ou do desejo sexual. Longe disso, a vida sexual não vai "acabando" com o passar dos anos, mas ainda é algo tão censurado, que, na maioria das vezes, os idosos se sentem inibidos de ter relações sexuais e até falar sobre sexo (3).

Destarte, este relato objetiva discorrer sobre a importância da promoção de saúde para o indivíduo idoso, além de estimular o debate acerca das vivências, expressões, impactos, prejuízos e dificuldades da sexualidade na terceira idade, buscando desmistificar estereótipos de uma velhice assexuada. Ademais, tentou-se criar, a partir da discussão do tema, uma maior abertura para que os idosos se sintam mais confortáveis em conversar com pessoas que possam os orientar com informações seguras acerca do assunto, resultando em uma melhor qualidade de vida (4).

\section{RELATO}

A ação "Sexualidade tem prazo de validade? Uma abordagem na terceira idade" (Figura 1) ocorreu no dia 25 de novembro de 2020, às 19 horas, com o fito de desmistificar um tema bastante negligenciado e, ainda, permeado de tabus e estigmas sociais. Nesse contexto, tendo em vista o potencial transformador do acesso à informação segura e ao conhecimento acerca da sexualidade na senescência, a palestra virtual foi previamente planejada considerando recortes temáticos indispensáveis, dentro de uma abordagem interativa, responsável e sensível para com o público-alvo, estudantes e profissionais da área da saúde e a população, sobretudo, idosa.

Para tanto, foram utilizados o Instagram e o WhatsApp como ferramentas de divulgação do evento e as inscrições foram realizadas por meio de um formulário eletrônico no Google Forms, em que o indivíduo, ao finalizá-lo, era direcionado a um grupo no Whatsapp, criado com o intuito de promover um contato mais direto com os participantes, além de proporcionar um canal comunicativo para sanar dúvidas após a palestra. 
Figura 1. Imagem da divulgação da ação.

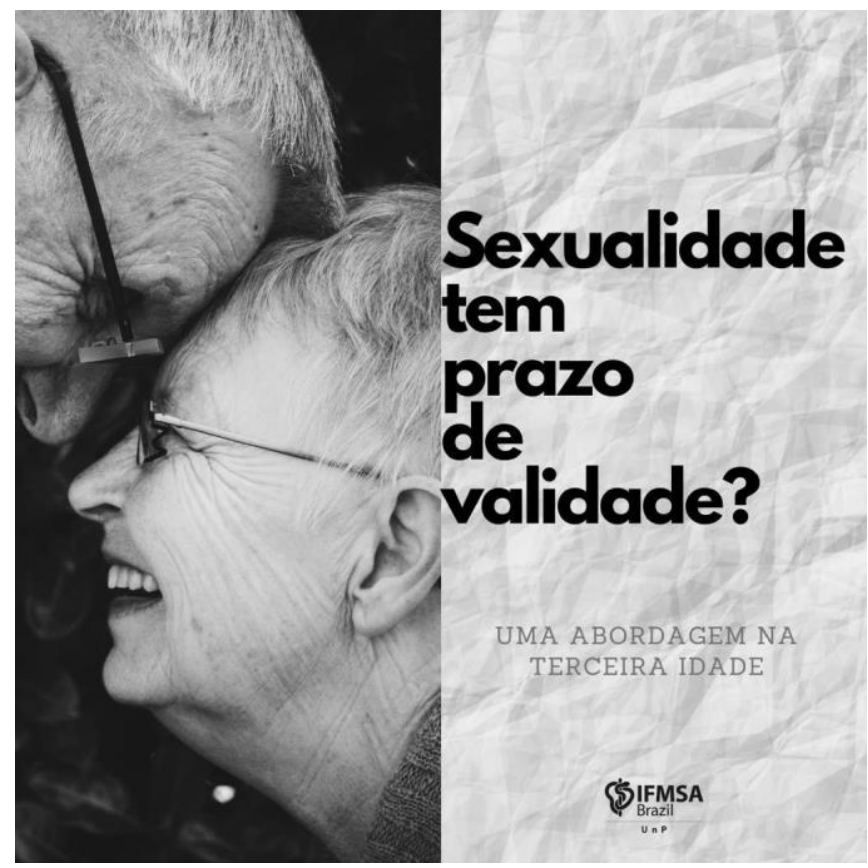

Fonte: Autoria própria.

Nessa perspectiva, a ação foi viabilizada pela plataforma digital Google Meet, em dois momentos distintos (Figura 2). No primeiro, uma especialista em fisioterapia pélvica abordou os tabus referentes à sexualidade da pessoa idosa e ao processo natural de envelhecimento, além de temas paralelos, como identidade de gênero e orientação sexual, infecções sexualmente transmissíveis, medidas de prevenção e envelhecimento saudável, valorizando, em um verdadeiro entrelace temático, discussões que vão sendo anuladas do convívio social à medida que a faixa etária dos indivíduos avança.

Figura 2. Imagem da divulgação das palestrantes da ação.

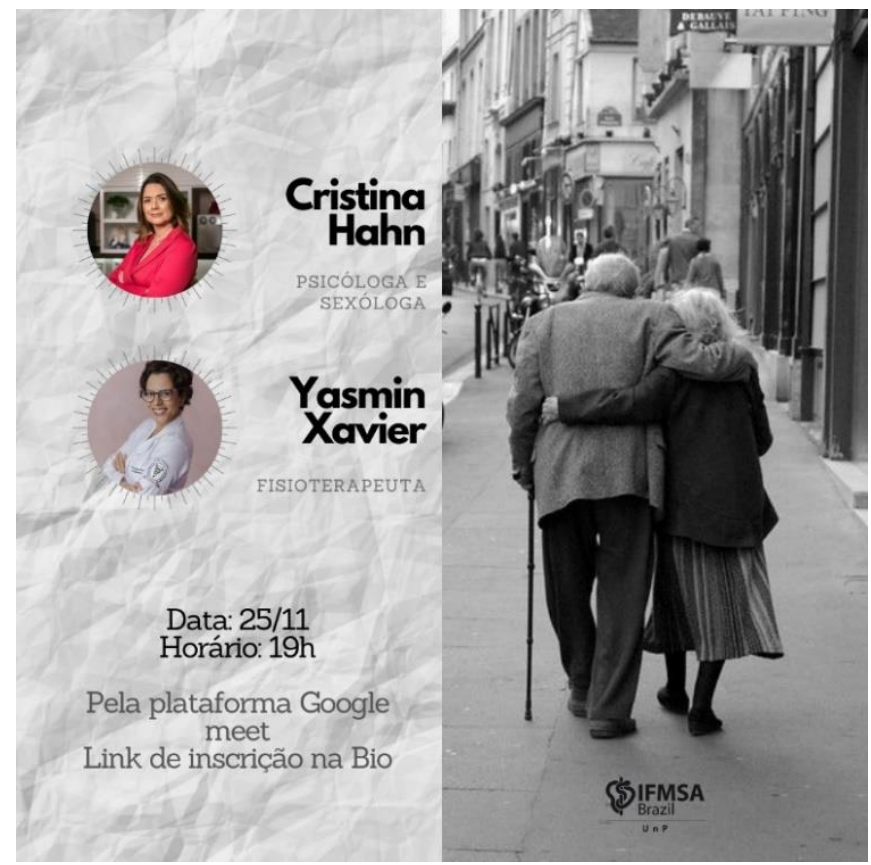

Fonte: Autoria própria.
Na sequência, uma sexóloga e psicóloga manejou os aspectos biológicos, sociais e psicológicos do idoso, associados à sexualidade, agregando pano de fundo substancial para que o debate seguisse fluido e instigante, permitindo um leque de reflexões e transformações de mentalidades. Além disso, corroborou, especialmente, aos que estão em formação acadêmica, maior sensibilidade na construção de um olhar atento, inclusivo e sem preconceitos no que tange à percepção da sexualidade dos mais velhos.

Ademais, os participantes, em roda de conversa, puderam esclarecer questionamentos junto às palestrantes e relatar vivências e experiências pessoais, tornando o "bate-papo" bastante enriquecedor, haja vista o debate proporcionado fomentar discussão essencial para a estruturação de novas perspectivas em educação e saúde direcionadas ao idoso, como o uso de preservativos e a prevenção primária contra IST's, suprimindo os estigmas do errado ou do proibido tão difundidos quando se trata da terceira idade.

Outrossim, é válido enfatizar o desafio de expor temáticas culturalmente estigmatizadas por meio de um evento virtual, o qual não possibilita o olho no olho e o sentir a real troca de saberes entre os envolvidos. Por outro lado, a atividade online permitiu reunir um público bastante diverso, de diferentes faixas etárias, níveis de escolaridade, áreas de atuação profissional e localidades e proporcionar uma troca de conhecimentos extremamente enriquecedora.

Em relação à análise de impacto, foi gerado um formulário previamente à execução da ação, com perguntas acerca da expressão da sexualidade na velhice. Posteriormente à palestra, outro questionário foi respondido pelo público, a fim de realizar um comparativo entre conhecimento prévio e adquirido com a atividade.

Com isso, pelos resultados obtidos, destacam-se o alcance à reflexão responsável e coletiva acerca da temática e a partilha de conhecimentos importantes e edificadores para a quebra de paradigmas ainda tão presentes na sociedade, preservando, sobretudo, a integralidade da pessoa idosa. (Figuras 1 e 2).

\section{DISCUSSÃO}

Em análise, a ação colocou em pauta os direitos sexuais do idoso, a promoção da saúde e liberdade individual, seguindo a tendência contemporânea dos estudos voltados para a terceira idade. Com isso, pode-se observar o contraste entre as gerações de participantes, perante a dualidade do prazer e do pudor, e na desmistificação das crenças populares limitantes.

Uma vez que foram abordados os aspectos sociais e fisiológicos do processo de envelhecimento, tornou-se claro o papel dos avanços na medicina na inversão da pirâmide etária brasileira. Segundo o Instituto Brasileiro de Geografia e Estatística (IBGE), a evolução dos métodos contraceptivos, a ampliação das campanhas de vacinação e a universalização do sistema público de saúde os principais responsáveis por essa mudança no perfil populacional e tem como consequência, a necessidade de um maior direcionamento de estudos para entendimento das necessidades desse grupo. 
Entendeu-se também, que o envelhecer do ser humano trata-se de um processo de mudanças universais, porém geneticamente únicas, as quais são traduzidas na diminuição da plasticidade cerebral e, consequentemente, no aumento da vulnerabilidade, elevando as perdas fisiológicas, incluindo das atividades reprodutivas (6). Dessa forma, não houve intercorrências ao se tratar do nivelamento dos participantes da ação, já que as palestrantes cumpriram com o objetivo de introduzir de maneira sucinta e traçar o direcionamento dos assuntos abordados com excelência.

Entendeu-se também que as mudanças esperadas no processo de envelhecimento influenciam muito na resposta sexual dos idosos, pois é nessa fase em que há o início da menopausa nas mulheres, acarretando a diminuição dos hormônios ovarianos, sobretudo, às custas de estrogênios. $\mathrm{Na}$ vulva, há redução anatômica dos grandes lábios, da secreção das glândulas sebáceas e sudoríparas, bem como atrofia das glândulas de Bartholin. Além disso, têm-se o surgimento de dispareunia e sangramentos, em resposta à retração do intróito vaginal, tornando o ato sexual incômodo e traumático para a maioria delas. Já nos homens, apesar de não ter uniformidade nas transformações, de um modo geral, apresentam ereção mais flácida, sendo necessário mais tempo para alcançar o orgasmo, incidência aumentada de alterações na próstata, acarretando diversas manifestações relacionadas à manutenção da ereção, bem como surgimento de incontinência urinária (7). Dessa maneira, ambos sexos vivenciam, sob perspectivas diferentes, muitos desafios no que diz respeito à relação com o próprio corpo e o que isso representa para a sociedade, sendo fundamental a compreensão da sexualidade como um processo amplo, não vinculado somente à apreciação de corpos jovens, performance sexual e penetração, mas sim um movimento fluido, pautado na compreensão, conexão e acolhimento das marcas do tempo. Portanto, todo conhecimento compartilhado durante a palestra teve intenção de reverberar a proteção e o cuidado nos ciclos sociais dos participantes, a fim de proteger e entender as limitações desse recorte populacional.

Além disso, assuntos como autoestima e as mudanças biopsicossociais misturaram-se com relatos e experiências compartilhadas agregando valor de acolhimento e proximidade entre os conteúdos e a vida pessoal dos participantes. As alterações físicas, acarretadas pelo passar dos anos, devem ser respeitadas e vistas com carinho por se tratar de algo natural. Isso posto, as adaptações devem ser bem-vindas com a busca pelo conforto e apreciação dessa nova fase (5).

Por conseguinte, a atenção ao Prisma da Sexualidade na terceira idade é um tópico relevante não apenas para os estudantes da área da saúde, mas sim para toda população. Ao passo que se discute sobre proteção e visibilidade da vida sexual dos idoso, os tabus referentes ao assunto são quebrados, permitindo o cumprimento do direito à saúde e qualidade de vida, possibilitando o exercício do amor-próprio e da socialização na faixa etária crescente do Brasil. Finalmente, apesar do longo caminho para suprir a lacuna do acesso a essas informações, cada grupo atingido representa uma importante parte do processo de levar educação sexual e visibilidade para as diretrizes da sexualidade para os idosos.

\section{CONCLUSÃO}

Dessa maneira, o projeto proporcionou aos participantes, um canal aberto para discussões sobre a sexualidade do idoso, sob uma perspectiva mais integrativa, acolhedora e empática, trazida por profissionais capacitados e experientes, não havendo grandes limitações a respeito de nivelamento, perante os participantes em relação aos conteúdos ministrados. Além disso, trouxe à superfície as principais problemáticas relacionadas ao tema, tais quais os estereótipos atrelados ao comportamento dessa faixa etária e a dificuldade ao acesso às informações de saúde, sobretudo, no que diz respeito ao processo de envelhecimento e suas dinâmicas de vivência e expressão da sexualidade. Nesse contexto, a ação serviu como uma experiência enriquecedora para cada um dos participantes envolvidos, que certamente carregam consigo uma visão mais abrangente e desmistificada sobre a temática e poderão aplicá-la em seu cotidiano.

\section{CONFLITO DE INTERESSE}

Os autores declaram não haver qualquer potencial conflito de interesse relacionado ao presente artigo.

\section{FINANCIAMENTO}

Não foram utilizados recursos financeiros nesta produção científica.

\section{REFERÊNCIAS}

1. Almeida T, Lourenço ML. Envelhecimento, amor e sexualidade: utopia ou realidade? Rev Bras Geriatr Gerontol. 2007 Jan-Abr;10(1):101-14.

2. Barros TAF, de Assunção ALA, do Carmo Kabengele D. SEXUALIDADE NA TERCEIRA IDADE: SENTIMENTOS VIVENCIADOS E ASPECTOS DE INFLUÊNCIA. CBIOS. 2020 Abr;6(1):47-47.

3. Rozendo AS, Alves JM. Sexualidade na terceira idade: tabus e realidade. Rev Kairos. 2015 Set;18(3):95-107.

4. Silva NRN. Sexualidade na Velhice: a Visão do Idoso e os Fatores Influenciadores [Trabalho de Conclusão de Curso em Terapia Ocupacional]. Brasília: Universidade de Brasília; Faculdade de Ceilândia; 2014. 31 p.

5. Hogan R. Human sexuality - a nursing perspective. ( $2^{\underline{a}}$ ed.). Connecticut: Appleton Century Crofts; 1984. 558 p.

6. Vieira KFL, Coutinho MPL, Saraiva ERA. A Sexualidade $\mathrm{Na}$ Velhice: Representações Sociais De Idosos Frequentadores de Um Grupo de Convivência. Psicol Ciênc Prof. 2016 Jan-Mar;36(1):196-209. 
7. Alencar DL, Marques APO, Leal MCC, Vieira JCM. Fatores que interferem na sexualidade de idosos: uma revisão integrativa. Cien Saude Colet. 2014 Abr;19(8):3533-42. 